\title{
The 2014 San Antonio Breast Cancer Symposium: A successful lift-off for breast immunotherapy?
}

\author{
David B Page ${ }^{1}$, Jarushka Naidoo ${ }^{1}$ and Heather L McArthur ${ }^{1}$
}

Therapeutic immune checkpoint antibodies promote potentially durable cancer control by modulating key regulatory factors of the endogenous anti-tumor immune response. The first clinical trial data of these agents in breast cancer were presented at the 2014 San Antonio Breast Cancer Symposium, with seemingly modest response rates compared with metastatic melanoma and Hodgkin's lymphoma. In this article, we review the San Antonio immunotherapy data, drawing key analogies to historical experiences in metastatic melanoma that support an enthusiastic outlook for immunotherapy in breast cancer.

npj Breast Cancer (2015) 1, 15001; doi:10.1038/npjbcancer.2015.1; published online 2 June 2015

\section{INTRODUCTION}

Modern immunotherapy has markedly transformed the treatment landscape for metastatic melanoma as evidenced by the recent rapid-fire food and drug administration (FDA) approval of three immunologic 'checkpoint antibodies' (ipilimumab, pembrolizumab, and nivolumab). These checkpoint antibodies function by binding and blocking key immune regulatory proteins, cytotoxic T-lymphocyte antigen 4 (CTLA4), ${ }^{1}$ programmed death 1 (PD-1), or programmed death ligand 1 (PD-L1), ${ }^{2}$ thereby 'releasing the brakes' on lymphocytes and permitting a robust immune response against the tumor. The most recently approved antibody, nivolumab, produced a $40 \%$ durable objective response rate and a near-doubling of 1-year overall survival compared with conventional chemotherapy. ${ }^{3}$

In light of these impressive results, investigators have been keen to evaluate immune checkpoint antibodies in other tumor types. The clinical activity of anti-PD-1/L1 therapy was recently reported across a growing list of malignancies, including nonsmall cell lung cancer, renal cell carcinoma, gastric cancer, Hodgkin's lymphoma, and bladder cancer. To date, limited clinical trial data have been presented for breast cancer. However, recent observations that tumor-infiltrating lymphocytes (TILs) are both prognostic and predictive supports the hypotheses that the immune system is implicated in the biology of breast cancer, mediates favorable outcomes, and that immunotherapy may be used as a rational therapeutic modality to enhance outcomes.

The emerging role of immunotherapy in breast cancer was evidenced by a number of presentations at this year's San Antonio Breast Cancer Symposium (SABCS). First, retrospective analyses of TILs provided additional evidence that anti-tumor immunity mediates survival in breast cancer. ${ }^{4}$ Next, the first unequivocal cases of objective response to immune checkpoint blockade in metastatic breast cancer were reported from two independent phase I clinical trials with anti-PD-1/L1 therapy. ${ }^{5,6}$ Finally, preliminary data were presented illustrating how novel combination strategies-such as immune checkpoint blockade with tumor cryoablation-might be used to enhance response to immuno- therapy in breast cancer. ${ }^{7}$ Herein, we review the developments to date and potential future directions for immunotherapy in breast cancer.

\section{THE TIL STORY: REPEATED CREDENCE FOR BREAST IMMUNOTHERAPY}

In 2013, leaders in the field convened at the first annual San Antonio TILs working group to develop a standardized metric by which to measure TILs using hematoxylin and eosin (H\&E). ${ }^{8}$ Using these consensus guidelines, Dr Edith Perez presented a retrospective analysis of TILs in patients with HER2-positive early-stage breast cancer who received adjuvant chemotherapy $+/-$ trastuzumab in the ECOG N9831 trial at SABCS 2014. ${ }^{4}$ Two key findings were presented, one confirmatory and the other novel. The confirmatory finding, illustrated previously in several adjuvant data sets, ${ }^{9,10}$ was that TIL quantity by H\&E is prognostic, with increasing stromal TILs correlating with improved outcome following chemotherapy both as continuous and binary variables. ${ }^{4}$ These data suggest that anti-tumor immunity contributes toward cure in early-stage breast cancer, and provides a compelling rationale to investigate immunotherapy in early-stage breast cancer, particularly in patients with low TILs and a high risk of relapse.

The novel finding was that the prognostic association was less apparent in trastuzumab-treated patients. From the previously reported FinHer study of chemotherapy with or without a shorter duration of trastuzumab, ${ }^{11}$ stromal TILs as a continuous variable was associated with improved outcome following trastuzumab. The N9831 analysis, however, failed to confirm this finding. The FinHer data set also suggested that TIL-high tumors benefited to a greater degree from trastuzumab, in contrast to the N9831 data set that demonstrated TIL-low tumors benefited from trastuzumab and TIL-high tumors fared equally well, with or without trastuzumab.

One way to come to terms with these discrepancies is to conceptually 're-classify' trastuzumab as a bona fide immunotherapy, which is supported by preclinical observations that

${ }^{1}$ Memorial Sloan Kettering Cancer Center, Breast Medicine Service, New York, NY, USA

Correspondence: DB Page (paged@mskcc.org)

Received 21 January 2015; accepted 21 January 2015 
trastuzumab activity is mediated by both innate (antigen nonspecific) and adaptive (antigen-specific) immunity. ${ }^{12}$ As such, the first discrepancy-that TILs are less prognostic in trastuzumabtreated patients-could be explained by the possibility that trastuzumab serves to immunologically rescue 'low-TIL' tumors, thereby improving their prognosis and diminishing the importance of baseline TIL density. The second discrepancy-that 'highTIL' tumors did not benefit from the addition of trastuzumab in the N9831 data set-is likely explained by the excellent prognosis of these patients irrespective of trastuzumab, potentially because of pre-existing anti-tumor immunity. While the FinHer data set demonstrated benefit with trastuzumab in the high-TIL subgroup, the subgroup was small $(n=22)$ with only six events across two arms. So given this data, the 'take-home' message from N9831 is that the 'high-TIL' patient-characterized by excellent baseline tumor immunogenicity-fares well with standard-of-care chemotherapy alone, whereas patients with lower TIL quantities benefit from the addition of trastuzumab, with the caveat that these findings are discordant with those from the smaller FinHer study. A meta-analysis of the data from all the pivotal adjuvant chemotherapy/trastuzumab trials would further refine these observations and may further delineate which patients could potentially benefit from modern immunotherapy strategies.

\section{THE POWER OF THE ANECDOTE: FROM COLEY'S TOXIN TO PEMBROLIZUMAB}

More than a century ago, a surgeon named William Coley noticed that one of his cancer patients experienced a remission after a serious infection. With further investigation he learned that other physicians had observed similar responses. He consequently developed 'Coley's toxin,' a cocktail of bacterial products that could induce marked tumor regressions when injected into sarcomas, albeit with somewhat inconsistent results, and with the unfortunate side effect of sepsis in some. ${ }^{13}$ With these efforts, he is largely credited as being the founder of cancer immunotherapy. More than a century later, initial experiments of ipilimumab in metastatic melanoma were remarkably similar to the Coley's toxin experience, characterized by frequent toxicities (including life-threatening immune colitis) and infrequent objective responses $(10.9-15.2 \%) .{ }^{14,15}$ Akin to William Coley, modern immunotherapists used anecdotes of complete responses, delayed responses, and durable stable disease to sustain enthusiasm for modern immune checkpoint blockade strategies. ${ }^{16}$ Thanks to these unwavering efforts, ipilimumab was ultimately FDA approved in metastatic melanoma on the basis of durable survival benefit, with many ipilimumab-treated patients surviving free of disease for more than 10 years. ${ }^{14,15}$ Furthermore, we have learned, through experience, how to safely manage the toxicities of ipilimumab.

In some ways, this year's initial reports of immune checkpoint blockade in breast cancer echo these historical experiences. Reports from a phase I study of MPDL3280A, an anti-PD-L1 antibody, ${ }^{6}$ and a phase I study of pembrolizumab, an anti-PD-1 antibody, in triple-negative breast cancer ${ }^{5}$ were modest, with media headlines emphasizing that the objective response rates in breast cancer (33 and 19\%) paled in comparison with melanoma $(40 \%)^{3}$ and Hodgkin's lymphoma $(87 \%) .{ }^{17}$ However, if we draw analogies to melanoma experience with ipilimumab, we have reason to remain optimistic. The first similarity to ipilimumab in melanoma is that objective responses to anti-PD1/PD-L1 therapy in breast cancer appeared to be durable, with several patients treated for more than 11 months. Second, in addition to the reports of complete $(n=2)$ and partial $(n=6)$ responders, a notable proportion of patients experienced response kinetics similar to the melanoma population, with unconventional but durable clinical benefit seen in 8 patients $(26 \%, 11 \%)$ who achieved stable disease, and several with prolonged tumor regression after initial development of new lesions. In melanoma, we know that many long-term survivors, who received immune checkpoint blockade, develop new lesions that are successfully managed with radiation or surgery without additional systemic therapy. ${ }^{18}$ These observations provide hope that survival benefit in breast cancer may exceed the apparent benefit using conventional response criteria

Furthermore, in contrast to Coley's toxins and the early ipilimumab experience, both MPDL3280A and pembrolizumab were safe and well tolerated. ${ }^{5,6}$ Minimal grade 3-4 adverse events were reported (MPDL3280A: 8\% adrenal insufficiency; pembrolizumab: $3 \%$ anemia, headache, aseptic meningitis, pyrexia, and decreased fibrinogen). One death occurred in the pembrolizumab trial; however, this death was attributed to disseminated intravascular coagulation, which is not generally observed with anti-PD-1 therapy. The toxicity profiles reported at SABCS 2014 echo the larger body of clinical evidence that anti-PD-1/PD-L1 is extremely safe and may provide clinical activity while preserving quality of life in heavily pre-treated patients.

\section{PERSONALIZED IMMUNOTHERAPY: THE FUTURE IN BREAST CANCER}

Despite our calls for optimism, these trials also suggest that a 'onesize-fits-all' immunotherapy approach may not succeed across tumor types, or across breast cancer subtypes. For example, both of the aforementioned anti-PD-1/L1 antibody phase I breast cancer trials excluded patients with PD-L1-negative tumors. Because PD-L1 positivity is likely to enrich for response, an anti-PD-1/L1 trial in an unselected population might achieve even fewer objective responses than reported. However, the significance and definition of PD-L1 positivity remains highly controversial. It was originally believed that tumors expressing PD-L1 would be most likely to respond to anti-PD1/PD-L1 therapy. ${ }^{19}$ However, recent data suggest that PD-L1 positivity of infiltrating immune cells is better associated with response, and that PD-L1 may be a marker of prior T-cell activation rather than tumor immune escape. ${ }^{20,21}$

However, PD-L1 expression alone, or TIL quantity by H\&E staining alone, is unlikely to adequately predict response to immunotherapy. More nuanced studies of TILs may provide an understanding of the mechanisms of immune resistance in breast cancer, and may guide rational therapeutic targeting of molecules implicated in tumor immune evasion and escape. A recent analysis of baseline tumor biopsies preceding anti-PD-L1 therapy serves to illustrate this concept. ${ }^{20}$ Tumors that responded to anti-PD-L1 therapy were generally characterized by high-TIL density and PDL1 positivity. In contrast, tumors that progressed following PD-L1 therapy were characterized by one of several profiles: some 'immunologically ignorant' tumors exhibited low-TIL density and PD-L1 negativity; other 'immunologically non-functional' tumors contained more TILs but were PD-L1-negative; finally, a third group of 'immunologically excluded' tumors contained dense, PD-L1-positive lymphocytes at the leading edge of the tumor, but with no intratumoral TILs. While these findings have not yet been validated in larger studies, they highlight the principle that a singular biomarker may be inadequate to predict response to immunotherapy. Furthermore, the lack of a standardized assay for PD-L1 and the dynamic nature of PD-L1 expression remain ongoing challenges in this field.

How can we forge ahead to optimize immunotherapy in breast cancer? First, as a field, we must acknowledge and embrace the complexity of immunology. Despite the prognostic considerations of TILs, the TIL is not a singular entity: TILs comprises multiple cell types, some capable of killing tumor (and protecting from relapse) and others capable of promoting tumor growth. Similarly, immunotherapy is not a singular entity: aside from anti-PD-1/L1, dozens of other immunotherapeutic targets are being evaluated in phase I studies, many of which have strong pre-clinical rationale in breast cancer. ${ }^{22}$ For example, some breast cancers, particularly 


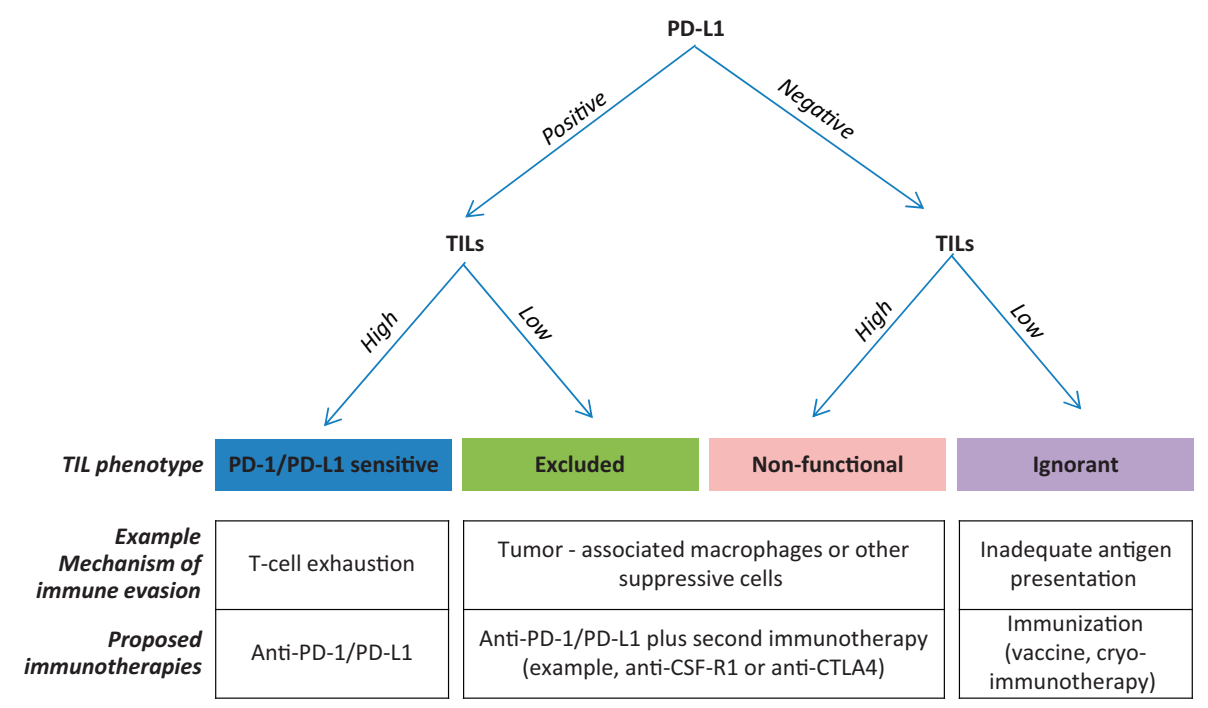

Figure 1. Conceptualization of how multiple immunologic biomarkers could be used to personalize immunotherapy. CSF-1R, colonystimulating factor 1 receptor; PD-1, programmed death 1; PD-L1, programmed death ligand 1; TILs, tumour-infiltrating lymphocytes.

the cancers that are PD-L1-positive but resistant to anti-PD-1/L1, might develop immune resistance by recruiting tumor-associated macrophages (TAMs) - suppressive macrophages that are associated with adverse prognosis in breast cancer. ${ }^{23}$ Antibodies targeting colony-stimulating factor 1 receptor, a growth factor receptor for TAMs, are undergoing phase I evaluation and may ultimately be effective in overcoming this resistance mechanism. For these reasons, we must expand our efforts beyond the H\&E TIL biomarker and anti-PD-1/PD-L1 therapeutic antibodies.

One potential way forward is to integrate independently validated immune-based biomarkers, and to use these data to personalize immunotherapy using a variety of novel agents and combination therapies. For example, if we assume that PD-L1 expression and TIL quantity are binary variables, we can conceive of four distinct immune phenotypes, each which may benefit from a distinct immunotherapeutic strategy (Figure 1). While the TIL-high, PD-L1 positive tumors may respond to anti-PD-1/L1 therapy alone, other tumors may require novel agents or combination approaches. Perhaps the 'immunologically ignorant' cancers-with minimal or no TILs or PD-L1-might benefit from an immunization strategy to induce a de novo immune response against the tumor and promote influx of TILs. One such immunization strategy employs tumor cryoablation plus checkpoint antibody to generate tumor antigen release followed by T-cell activation. In a pilot clinical study presented at SABCS, treatment with cryoablation plus ipilimumab led to production of interferon gamma and proliferation of T-cell clones. ${ }^{7}$ Because interferon gamma is associated with upregulation of PD-L1, cryoimmunotherapy might be particularly suitable for the 'immunologically ignorant' tumors that exhibit low TILs and PD-L1 negativity at baseline.

While anti-PD-1/PD-L1 alone may not be a 'one-size-fits-all' drug for breast cancer, combinatorial strategies and novel agents might make all tumors-TIL low or TIL high, PD-L1 negative or PD-L1 positive-amenable to an immunotherapeutic approach. In summary, while fraught with challenges, breast cancer immunotherapy has 'lifted-off,' and in our opinion, is here to stay.

\section{COMPETING INTERESTS}

The authors declare no conflict of interest.

\section{FUNDING}

The authors declare that no funding was received.

\section{REFERENCES}

1 Krummel MF, Allison JP. CD28 and CTLA-4 have opposing effects on the response of T cells to stimulation. J Exp Med 1995; 182: 459-465.

2 Freeman GJ, Long AJ, Iwai Y, Bourque K, Chernova T, Nishimura $\mathrm{H}$ et al. Engagement of the PD-1 immunoinhibitory receptor by a novel B7 family member leads to negative regulation of lymphocyte activation. J Exp Med 2000; 192: 1027-1034.

3 Robert C, Long GV, Brady B, Dutriaux C, Maio M, Mortier L et al. Nivolumab in previously untreated melanoma without BRAF mutation. N Engl J Med 2015; 372: 320-330.

4 Perez EA, Ballman KV, Anderson SK, Thompson EA, Badve SS, Bailey $\mathrm{H}$ et al. Stromal tumor-infiltrating lymphocytes(S-TILS): In the alliance N9831 trial S-TILs are associated with chemotherapy benefit but not associated with trastuzumab benefit. San Antonio Breast Cancer Symposium: San Antonio, TX, 2014, December 8-13, 2014.

5 Nanda R, Chow LQ, Dees EC, Berger R, Gupta S, Geva R et al. A phase lb study of pembrolizumab (MK-3475) in patients with advanced triple-negative breast cancer. San Antonio Breast Cancer Symposium: San Antonio, TX, 2014, December 8-13, 2014.

6 Emens LA, Braiteh FS, Cassier PA, Delord J, Eder JP, Shen X et al. Inhibition of PD-L1 by MPDL3280A leads to clinical activity in patients with metastastic triple-negative breast cancer. 2014 San Antonio Breast Cancer Symposium: San Antonio, TX, 2014, December 8-13, 2014.

7 Page DB, Yuan J, Diab A, Dong Z, Ginsberg A, Wong P et al. Integrated immunologic assessment of tumor infiltrating lymphocytes (TILs) and peripheral blood to assess synergy of cryoablation (cryo) plus ipilimumab (ipi) in early stage breast cancer (ESBC) patietns (pts). 2014 San Antonio Breast Cancer Symposium: San Antonio, TX, 2014, December 8-14, 2014.

8 Salgado R, Denkert C, Demaria S, Sirtaine N, Klauschen F, Pruneri G et al. Harmonization of the evaluation of tumor infiltrating lymphocytes (TILs) in breast cancer: recommendations by an international TILs-working group 2014. Ann Oncol 2015; 26: 259-271.

9 Loi S, Michiels S, Salgado R, Sirtaine N, Jose V, Fumagalli D et al. Tumor infiltrating lymphocytes is prognostic and predictive for trastuzumab benefit in early breast cancer: results from the FinHER trial. Ann Oncol 2014; 25: 1544-1550.

10 Adams S, Gray RJ, Demaria S, Goldstein L, Perez EA, Shulman LN et al. Prognostic value of tumor-infiltrating lymphocytes in triple-negative breast cancers from Two Phase III Randomized Adjuvant Breast Cancer Trials: ECOG 2197 and ECOG 1199. J Clin Oncol 2014; 32: 2959-2966.

11 Loi S, Michiels S, Salgado R, Sirtaine N, Jose V, Fumagalli D et al. Tumor infiltrating lymphocytes (TILs) indicate trastuzumab benefit in early-stage HER2-positive breast cancer. San Antonio Breast Cancer Symposium, 2013, December 10-14, San Antonio, TX, 2013. 
12 Park S, Jiang Z, Mortenson ED, Deng L, Radkevich-Brown O, Yang X et al. The therapeutic effect of anti-HER2/neu antibody depends on both innate and adaptive immunity. Cancer Cell 2010; 18: 160-170.

13 Coley WB. The Treatment of inoperable sarcoma by bacterial toxins (the mixed toxins of the Streptococcus erysipelas and the Bacillus prodigiosus). Proc $R$ Soc Med 1910; 3: 1-48.

14 Robert C, Thomas L, Bondarenko I, O'Day S, Weber J, Garbe C et al. Ipilimumab plus dacarbazine for previously untreated metastatic melanoma. $N$ Engl J Med 2011; 364: 2517-2526.

15 Hodi FS, O'Day SJ, McDermott DF, Weber RW, Sosman JA, Haanen JB et al. Improved survival with ipilimumab in patients with metastatic melanoma. $N$ Engl $J$ Med 2010; 363: 711-723.

16 Wolchok JD, Hoos A, O'Day S, Weber JS, Hamid O, Lebbe C et al. Guidelines for the evaluation of immune therapy activity in solid tumors: immune-related response criteria. Clin Cancer Res 2009; 15: 7412-7420.

17 Armand P, Ansell SM, Lesokhin AM, Halwani A, Millenson MM, Schuster SJ et al. Nivolumab in Patients with Relapsed or Refractory Hodgkin Lymphoma-Preliminary Safety, Effiacy, and Biomarker Results of a Phase I Study. 56th ASH Annual Meeting and Exposition; 2014 December 6-9, 2014; San Francisco, CA.

18 Page DB, Naidoo J, Momtaz P, Bogatch K, Kuk D, Panageas K et al. Patterns of longterm survival following ipilimumab (Ipi): the Memorial Sloan Kettering Cancer Cetner 10-year metastatic melanoma (MM) experience. SITC 2014: National Harbor, MD 2014, November 7-9, 2014
19 Topalian SL, Hodi FS, Brahmer JR, Gettinger SN, Smith DC, McDermott DF et al. Safety, activity, and immune correlates of anti-PD-1 antibody in cancer. $N$ Engl J Med 2012; 366: 2443-2454.

20 Herbst RS, Soria JC, Kowanetz M, Fine GD, Hamid O, Gordon MS et al. Predictive correlates of response to the anti-PD-L1 antibody MPDL3280A in cancer patients. Nature 2014; 515: 563-567.

21 Gros A, Robbins PF, Yao X, Li YF, Turcotte S, Tran E et al. PD-1 identifies the patient-specific $\operatorname{CD} 8(+)$ tumor-reactive repertoire infiltrating human tumors. J Clin Invest 2014; 124: 2246-2259.

22 Galluzzi L, Vacchelli E, Bravo-San Pedro JM, Buque A, Senovilla L, Baracco EE et al. Classification of current anticancer immunotherapies. Oncotarget 2014; 5: 12472-12508.

23 Ding ZC, Lu X, Yu M, Lemos H, Huang L, Chandler P et al. Immunosuppressive myeloid cells induced by chemotherapy attenuate antitumor CD4+ T-cell responses through the PD-1-PD-L1 axis. Cancer Res 2014; 74: 3441-3453.

(i) $\Theta$ This work is licensed under a Creative Commons Attribution cc. NonCommercial-NoDerivatives 4.0 International License. The images or other third party material in this article are included in the article's Creative Commons license, unless indicated otherwise in the credit line; if the material is not included under the Creative Commons license, users will need to obtain permission from the license holder to reproduce the material. To view a copy of this license, visit http:// creativecommons.org/licenses/by-nc-nd/4.0/ 